\title{
Evidence of Potential Averaging over the Finite Surface of a Bioelectric Surface Electrode
}

\author{
J. P. van Dijk, ${ }^{1}$ M. M. Lowery, ${ }^{2}$ B. G. Lapatki, ${ }^{1,3}$ and D. F. Stegeman ${ }^{1,4}$ \\ ${ }^{1} 920$ Department of Neurology/Clinical Neurophysiology, Donders Institute for Brain, Cognition and Behavior, Center for \\ Neuroscience, Radboud University Nijmegen Medical Center, P.O. Box 9101, 6500 HB Nijmegen, The Netherlands; ${ }^{2}$ School of \\ Electrical, Electronic and Mechanical Engineering, University College Dublin, Dublin, Ireland; ${ }^{3}$ Department of Orthodontics, \\ School of Dental Medicine, University of Freiburg i. Br., Freiburg, Germany; and ${ }^{4}$ Research Institute MOVE, Faculty of \\ Human Movement Sciences, VU University Amsterdam, Amsterdam, The Netherlands
}

(Received 20 November 2008; accepted 16 March 2009; published online 25 March 2009)

\begin{abstract}
Most bioelectric signals are not only functions of time but also exhibit a variation in spatial distribution. Surface EMG signals are often "summarized" by a large electrode. The effect of such an electrode is interpreted as averaging the potential at the surface of the skin beneath the electrode. We first introduce an electrical equivalent model to delineate this principle of averaging. Next, in a realistic finite element model of EMG generation, two outcome variables are evaluated to assess the validity of the averaging principle. One is the change in voltage distribution in the volume conductor after electrode application. The other is the change in voltage across the high impedance double layer between tissue and electrode. We found that the principle of averaging is valid, once the impedance of the double layer is sufficiently high. The simulations also revealed that skin conductivity plays a role. High-density surface EMG provided experimental evidence consistent with the simulation results. A grid with 120 small electrodes was placed over the thenar muscles of the hand. Electrical nerve stimulation assured a reproducible compound muscle response. The averaged grid response was compared with a single electrode matching the surface of the high-density electrodes. The experimental results showed relatively small errors indicating that averaging of the surface potential by the electrode is a valid principle under most practical conditions.
\end{abstract}

Keywords-Surface EMG, High-density, Finite element, Model, Muscle fiber.

\section{ABBREVIATIONS}

HD-sEMG High-density surface EMG

CMAP Compound muscle action potential

Address correspondence to J. P. van Dijk, 920 Department of Neurology/Clinical Neurophysiology, Donders Institute for Brain, Cognition and Behavior, Center for Neuroscience, Radboud University Nijmegen Medical Center, P.O. Box 9101, 6500 HB Nijmegen, The Netherlands. Electronic mail: h.vandijk@neuro.umcn.nl

\section{INTRODUCTION}

Bioelectric signals are not only a function of time but also have an often complex spatial distribution. However, in practical applications, it is common practice to summarize this spatial distribution. For example, in electrodiagnostic medicine, the spatial pattern of a surface EMG signal is often reduced to a single value using a large electrode over the skin. ${ }^{6,21,22}$ The implicit assumption is that the signal from such a large electrode approximates the average of the potential distribution beneath the electrode, or more precisely, how it would appear if the electrode were not present. This behavior is sometimes denoted as integration, ${ }^{12}$ a term that falsely suggests that the potential increases with increasing electrode size.

In the interpretation of experimental EMG data, and similarly in modeling studies, the action of a large electrode is described as averaging the potential over the surface of the skin., ${ }^{4,7-9,11}$ It should be noted, however, that such studies do not provide evidence to support this assumption, since the skin-electrode interface properties are not taken into account. In related application fields, the electrode has been modeled more precisely and the impedance between the electrode and the skin has been explicitly considered. ${ }^{2,13,20}$

Two essential assertions are implicit when assuming averaging of the surface potential at the electrode:

I. The distribution of the electric potential in the volume conductor, specifically at the skin surface under the electrode, is not altered by the presence of the electrode.

II. The signal at the electrode is the true average of the potential at the skin surface under the electrode.

Once the bioelectric electrode is applied to the skin, it forms an electrical double layer, the specific 


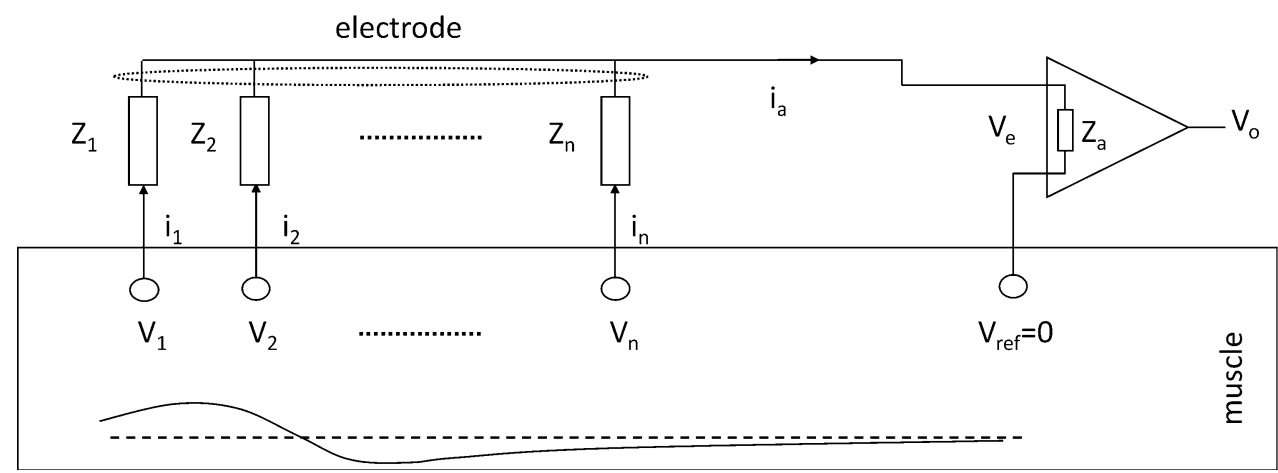

FIGURE 1. Schematic diagram of an EMG measurement using a single large electrode above muscle tissue. The spatially distributed potentials $(V)$, impedances $(Z)$, and current flow for a large electrode are shown. The amplifier with input impedance is visualized as well.

characteristics of which depend on the material of the electrode. ${ }^{24}$ The double layer can be modeled, however, with a series of $n$ parallel impedances, independent of the electrode material. From the schematic drawing in Fig. 1 of a single electrode overlying a muscle as bioelectric source, we can conclude for the relation between the currents $i_{k}$ and the electrode potential $V_{e}$ that

$$
\sum_{k=1}^{n} i_{k}=\sum_{k=1}^{n} \frac{V_{k}-V_{e}}{Z_{k}}
$$

where $Z_{k}$ is the $k$ th parallel impedance of the double layer, $i_{k}$ is the current through it, and $V_{k}$ is the potential at the skin surface beneath $Z_{k}$. From this

$$
V_{e} \sum_{k=1}^{n} \frac{1}{Z_{k}}=\sum_{k=1}^{n} \frac{V_{k}}{Z_{k}}-\sum_{k=1}^{n} i_{k}
$$

As modern amplifier systems have high input impedances, it can be assumed that, $i_{\mathrm{a}}$, the sum of all currents flowing across the skin-electrode interface

$$
\sum_{k=1}^{n} i_{k}=i_{a} \approx 0
$$

Each of the currents $i_{k}$ that emerge from the tissue into the electrode at one site must, therefore, vanish again into the tissue at other sites. In that case Eq. (2) can be rewritten as

$$
V_{\mathrm{e}} \approx \frac{\sum_{k=1}^{n} \frac{V_{k}}{Z_{k}}}{\sum_{k=1}^{n} \frac{1}{Z_{k}}}
$$

An important condition for the validity of assertion II above may be derived directly from Eq. (4). Equation (4) illustrates that the averaging of the potential at the electrode depends on the relative distribution of the impedances $Z_{k} . V_{\mathrm{e}}$ is not a simple average, but a weighted average of the potential distribution, whereby the relative reciprocals of the distributed impedances are the weighting factors. It is crucial to note that the above relates to the relative difference between the distributed electrode impedances $Z_{k}$ and is irrespective of their absolute values. For the second assumption to be true, we must thus assume that all impedances are equal, $Z_{k}=Z_{o}$, after which it follows that

$$
V_{e} \approx \frac{\sum_{k=1}^{n} V_{k}}{n}
$$

The above schematic approach implicitly takes the first assumption into account. The potentials in the tissue $V_{1}, V_{2}, \ldots, V_{n}$ (Fig. 1) are assumed not to be affected by the presence of the electrode. To satisfy this assumption, the impedance of the electrode-skin contact must be much greater than the effective impedances within the volume conductor.

In the following, we first use the results of a finite element volume conductor model to provide a quantitative foundation to support the above introductory reasoning. Next, the basic theoretical model and the simulation results are compared to an experimental condition in which high-density surface EMG (HD-sEMG) and a large electrode were both used to study the thenar muscle group during electrical nerve stimulation.

\section{METHODS}

\section{Finite Element Model}

A four-layer finite element volume conductor model was constructed to simulate electric muscle activity within a limited volume conductor representing part of a limb. The volume conductor was modeled as a 44-mm radius cylinder (Fig. 2a), comprising an inner core of bone $(5 \mathrm{~mm})$, surrounded by cylindrically 
(a)

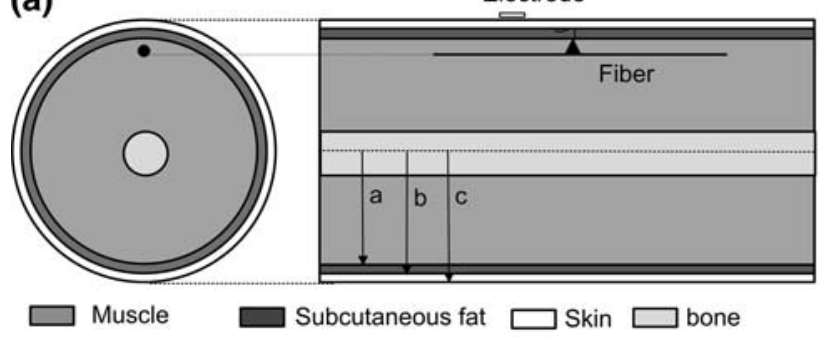

(b)

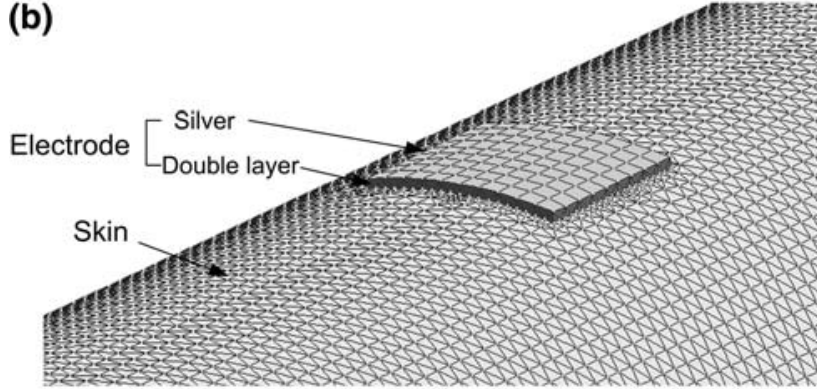

(c)

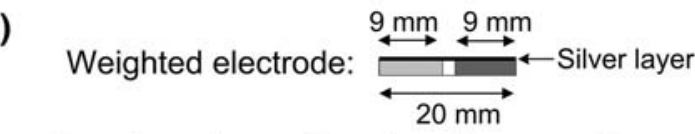

$\square$ Impedance A

Impedance B

$\checkmark$ Air

FIGURE 2. (a) Schematic diagram of the three-layer volume conductor model with an inner core of bone with $5 \mathrm{~mm}$ radius, $a=40 \mathrm{~mm}, b=43 \mathrm{~mm}$, and $c=44 \mathrm{~mm}$. (b) Surface of finite element model showing the thin double layer between the conductive electrode and the skin. (c) Schematic of weighted electrode used to illustrate the relative weighting of the surface potential by the electrode impedances. The double layer of the electrode shown in (b) was divided into two sections of different impedances, separated by a 2-mm air gap.

anisotropic muscle tissue $(35 \mathrm{~mm})$ and outer layers of subcutaneous fat tissue $(3 \mathrm{~mm})$ and skin tissue $(1 \mathrm{~mm})$. The model parameters are summarized in Table 1.

The finite element mesh was generated and the electrical potential throughout the model was solved using EMAS (EMAS 4.1, Ansoft Corp., Pittsburgh, PA), as described in previous studies. ${ }^{15}$ The volume conductor geometry was meshed using three-dimensional, linear, tetrahedral elements (Fig. 2b). The distance between adjacent nodes of the mesh varied from $0.1 \mathrm{~mm}$ along the muscle fiber to $8 \mathrm{~mm}$ deeper within the volume conductor. Wave propagation and inductive effects were assumed to be negligible, consistent with previous EMG models, and the electric field, $\vec{E}$, in the volume conductor was solved using Maxwell's equation derived from Ampere's Law,

$$
\nabla \cdot\left(\varepsilon \frac{\partial \vec{E}}{\partial t}+\sigma \vec{E}\right)=0
$$

where $\sigma$ is the conductivity and $\varepsilon$ is the permittivity of the medium. $\vec{E}$ is assumed to be a gradient field, i.e.,

TABLE 1. Model parameters (Conductivity and relative permittivity values were chosen at $100 \mathrm{~Hz}$ ).

Outer radius of cylinder

Cylinder length

Skin thickness

Subcutaneous fat thickness

Bone radius

Bone conductivity

Muscle conductivity (axial direction)

Muscle conductivity

(transverse direction)

Muscle relative permittivity (axial direction)

Muscle relative permittivity (transverse direction)

Fat conductivity

Fat permittivity

Skin conductivity (resistive skin)

Skin conductivity (conductive skin)

Skin permittivity

Double layer thickness

(numerically limited)

Default double layer conductivity

Default double layer relative permittivity

Electrode conductivity

$44 \mathrm{~mm}$
$300 \mathrm{~mm}$
$1 \mathrm{~mm}$
$3 \mathrm{~mm}$
$5 \mathrm{~mm}$
$0.02 \mathrm{~S} / \mathrm{m}$
$0.4 \mathrm{~S} / \mathrm{m}$
$0.09 \mathrm{~S} / \mathrm{m}$
$2 \times 10^{7}$
$4.4 \times 10^{6}$
$0.04 \mathrm{~S} / \mathrm{m}^{5}$
$1.5 \times 10^{5}$
$4.3 \times 10^{-4} \mathrm{~S} / \mathrm{m}$
$1.0 \mathrm{~S} / \mathrm{m}^{4}$
$5.53 \times 10^{4}$
$1 \mathrm{~mm}$
$3.33 \times 10^{-4} \mathrm{~S} / \mathrm{m}$
$\left(30 \mathrm{k} \Omega / \mathrm{cm}^{2}\right)$
$3.38 \times 10^{4}\left(30 \mathrm{nF} / \mathrm{cm}^{2}\right)$
$6 \times 10^{7} \mathrm{~S} / \mathrm{m}$

$\vec{E}=-\nabla \phi$, where $\phi$ is the electric scalar potential. It was assumed that the normal component of the electric field was equal to zero at the boundary of the model. The potential at the most distal node from the electrode was set to zero.

The muscle fiber action potential was represented as a line of 150 propagating point current sources, obtained by discretization of the second spatial derivative of the transmembrane potential and scaling appropriately. ${ }^{18}$ The muscle fiber action potential was simulated to propagate along the muscle fiber with uniform velocity in both directions away from the neuromuscular junction, which was located midway along the fiber. Charge balance was maintained during the initiation of the action potentials at the neuromuscular junction and extinction at the fibertendon transitions. ${ }^{15}$ In the examples presented, the muscle fiber was $150 \mathrm{~mm}$ in length, located $2 \mathrm{~mm}$ below the interface of the muscle and fat tissues and an action potential propagation velocity of $4 \mathrm{~m} / \mathrm{s}$ was assumed. The temporal evolution of the potential at the electrode, and throughout the model, was calculated at $0.25 \mathrm{~ms}$ intervals using the EMAS time domain solver.

As skin conductivity was found to be a critical parameter in the model, two different values of skin conductivity were incorporated in the model, denoted "resistive skin" and "conductive skin," to represent a large range of possible values (Table 1). The value for resistive skin was chosen based on values reported by 
Gabriel et al. ${ }^{10}$ while the conductive value is chosen based on Roeleveld et al. ${ }^{16}$

A single square electrode $(20 \mathrm{~mm} \times 20 \mathrm{~mm})$ was positioned on the skin surface directly above the muscle fiber (Fig. 2a, b), $33 \mathrm{~mm}$ away from the neuromuscular junction. The surface of the electrode was represented as a layer of conductive silver material using twodimensional $(1 \mathrm{~mm} \times 1 \mathrm{~mm})$ rectangular elements and a thin electrical double layer was included between the skin and electrode. The three-dimensional homogeneous double layer was realized by incorporating a uniformly thin $(1 \mathrm{~mm})$ layer between the conductive electrode surface and the skin tissue. The thickness of the double layer was chosen to preserve essential features of the double layer (a very thin layer of known resistance and capacitance), while maintaining the feasibility of meshing the area surrounding the electrode and minimizing the computational burden. The conductivity and relative permittivity of the double layer were calculated so that the electrode-skin impedance had a resistive component of $30 \mathrm{k} \Omega / \mathrm{cm}^{2}$ and a capacitive component of $30 \mathrm{nF} / \mathrm{cm}^{2}$, similar to experimentally reported values. ${ }^{17} \mathrm{~A}$ similar approach has previously been adopted in modeling the electrodeskin contact in impedance tomography ${ }^{13}$ and, more recently, for capturing microelectrode behavior. ${ }^{2}$

Simulated action potentials at the skin surface were examined, as the impedance of the double layer was varied, to evaluate the idealized concept of electrode averaging (Eq. 5, Fig. 1) over a range of double layer impedance values with the double layer conductivity and permittivity scaled proportional to one another.
To study the concept of weighted averaging (Eq. 4), in a separate study we divided the electrical double layer into two sections, impedance A and impedance B, separated by a small aperture as indicated in Fig. 2c. The impedances are connected by a thin, highly conductive silver layer, representing the electrode, spanning the surface across the top of both impedances. The impedance of the first section of the double layer was kept constant while the impedance of the second half was increased first by a factor of 2 and then 10 . The potential detected at the electrode surface was compared with that calculated based on weighting of the potential beneath each section of the electrode by the relative electrode impedances.

\section{High-Density Surface EMG}

To experimentally test the validity of the conditions for averaging, we compared high-density surface $E G^{1}$ with EMG data recorded using large surface electrodes placed over the same skin area. Two healthy subjects voluntarily participated in this part of the study. The study was approved by the local ethical committee of the Radboud University Nijmegen Medical Center, The Netherlands. We used flexible high-density electrode grids (Fig. 3a) and single large pregelled electrodes (Kendall-LTP H59P and H69P, Fig. $3 b$ ), cut to match with predefined electrode areas $\left(1 \times 2.6,1.8 \times 1.8,2.2 \times 2.2\right.$, and $\left.2.6 \times 3.4 \mathrm{~cm}^{2}\right)$.

The high-density electrode grid consisted of $8 \times 15$ $\mathrm{Ag}-\mathrm{AgCl}$ electrodes $(2 \mathrm{~mm}$ diameter) with an interelectrode distance of $4 \mathrm{~mm} .{ }^{14}$ It was placed on the (a)

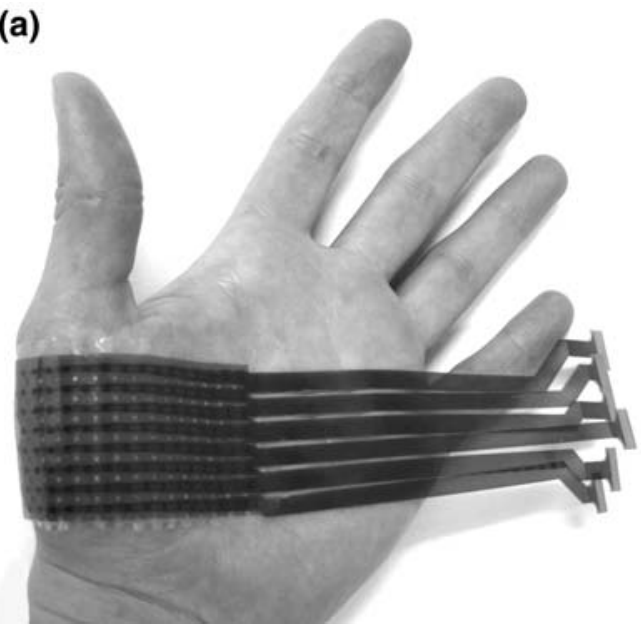

(b)

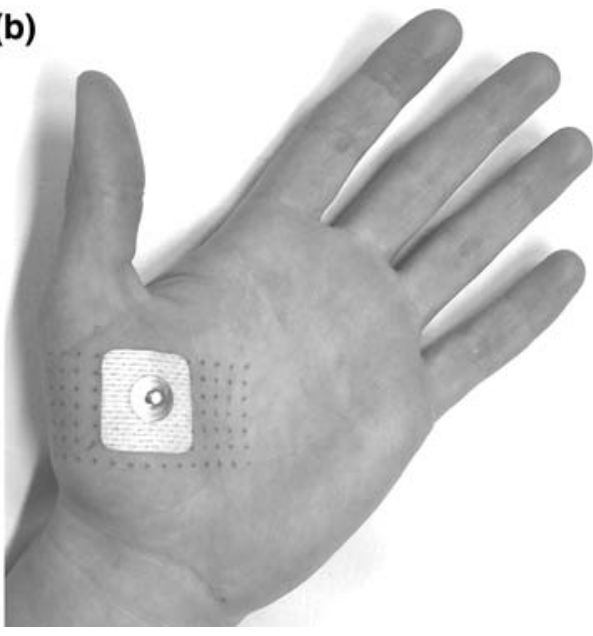

FIGURE 3. (a) The flexible grid of $120(8 \times 15)$ electrodes used for high-density surface EMG recordings. Interelectrode distance was $4 \mathrm{~mm}$ making the electrode $3 \times 5.8 \mathrm{~cm}^{2}$. The grid was placed transversally over the muscle belly and attached to the skin using double-sided adhesive tape with holes matching the electrode contacts. (b) Illustrates the position of one of the large electrodes. Note that the markers that are visible between the high-density electrodes are used to place the large electrode at the correct position. 
thenar muscle of the right hand (Fig. 3). The electrode grids were manufactured by Digiraster Tetzner $\mathrm{GmbH}$ (Stuttgart, Germany) and were fixed to the skin using double-sided adhesive tape. The tape contained holes matching the electrodes and had small holes in between, which were used to mark the electrode position using a permanent marker. A reference electrode was placed on the first metacarpophalangeal joint of the fifth digit for both recording situations. Data were recorded using a 130-channel BioSemi ActiveOne amplifier system (Amsterdam, The Netherlands) with a sample rate of $2048 \mathrm{~Hz}$.

To obtain a reproducible EMG signal, the median nerve was electrically stimulated using a constant current stimulator with fixed stimulation electrodes (pulse width $100 \mu \mathrm{s}$ ). By using supramaximal stimulation, all muscle fibers innervated by that nerve underneath the electrode were activated more or less simultaneously. This procedure yields reproducible results if the position of the hand is not altered and the temperature remains constant. The subject's thumb was immobilized and the temperature was maintained between 33 and $35^{\circ}$. The compound muscle action potential (CMAP) thus recorded was stored on disk and the electrode grid was removed. Next, the single large electrode was placed on a predefined location over the muscle. The prior applied markers guided the placement so that the single electrode matched the predefined area measured with HDsEMG as closely as possible (Fig. 3b). The smallest three electrodes were placed at three different positions over the muscle while the largest was placed on two different locations. For each recording a new pregelled electrode was used.

After removal of the stimulus artifact, the data were bandpass filtered (10-400 Hz) prior to further analysis. For each large electrode CMAP, an equivalent averaged CMAP was created by averaging the electric potential at the corresponding electrodes from the high-density electrode grid.

\section{Quantification of Error}

To quantify the difference between two time-varying signals for both the model simulation results and the experimental results, the percentage RMS error $\left(R M S_{\varepsilon}\right)$ was calculated as

$$
R M S_{\varepsilon}=100 \% \cdot \sqrt{\frac{\sum_{i}\left(\operatorname{signal}_{1}(i)-\operatorname{signal}_{2}(i)\right)^{2}}{\sum_{i} \operatorname{signal}_{1}^{2}(i)}}
$$

where $i$ denotes the sample points and signal $_{1}$ and signal $_{2}$ are the signals to be compared.

\section{RESULTS}

\section{Model Simulation Results}

The spatial and temporal variability of the results depend on parameters as conduction velocity, depth of the muscle fiber, and others. To illustrate this variability, Fig. 4 compares simulated single muscle fiber action potentials present at the skin surface beneath the electrode for the case of resistive (a) and conductive
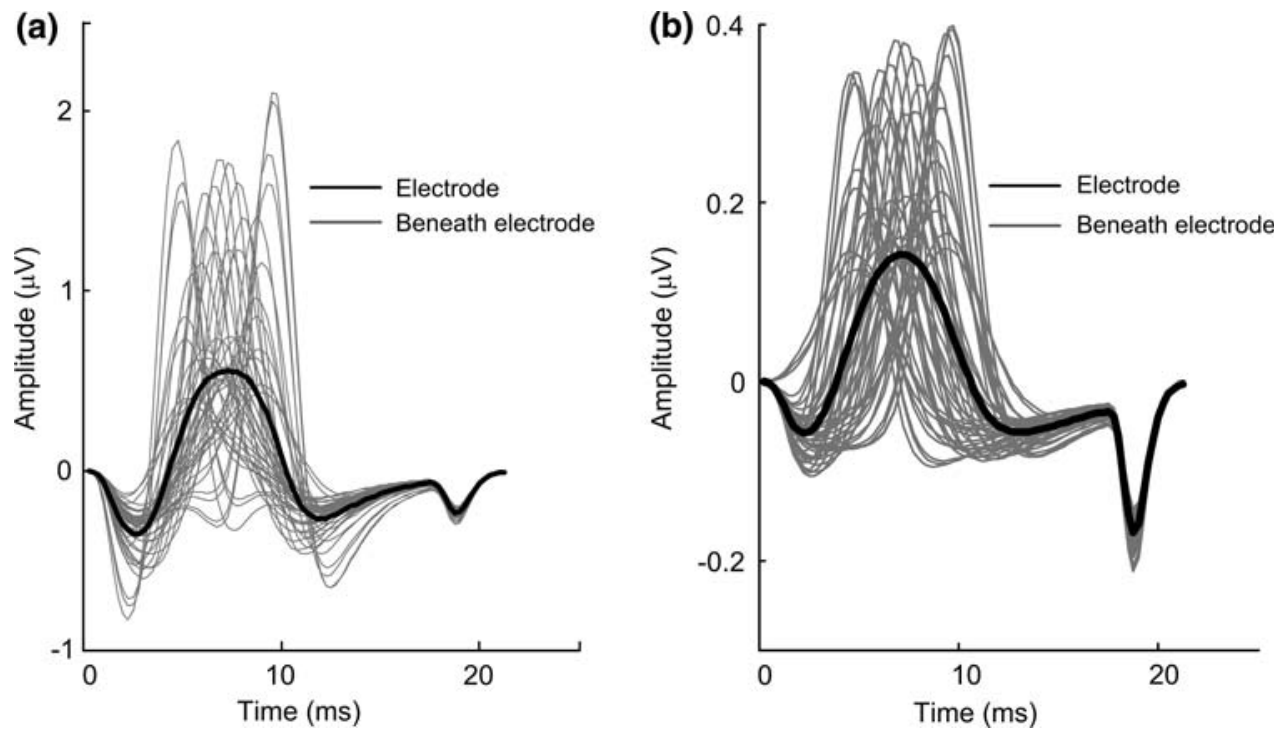

FIGURE 4. Simulated muscle fiber action potentials at the skin surface below the electrode (gray lines). A sample of 44 action potentials at various locations along the grid underneath the electrode is presented. The action potential detected at the electrode (metal surface) is also shown (thick black line). Results are presented for simulations conducted for both resistive (a) and conductive (b) skin tissue. 
skin (b) in the presence of a default electrical double layer (Table 1). A large spatiotemporal variability may be noted in the signals beneath the electrode. The potential predicted by the model at the electrode, equal across the entire electrode surface due to the low electrode resistance, is also presented.

In Fig. 5, the potential detected at the electrode is compared with the calculated average of the signal at the skin surface without the electrode present. At each time step, before the average potential was calculated, the two-dimensional potential distribution beneath the electrode was linearly interpolated with a spatial sampling of $0.01 \mathrm{~mm}$ to ensure that the average potential was not affected by the spatial sampling of the surface potential. A close agreement between the action potentials may be observed for resistive (Fig. 5a) and conductive (Fig. 5b) skin. The effect of
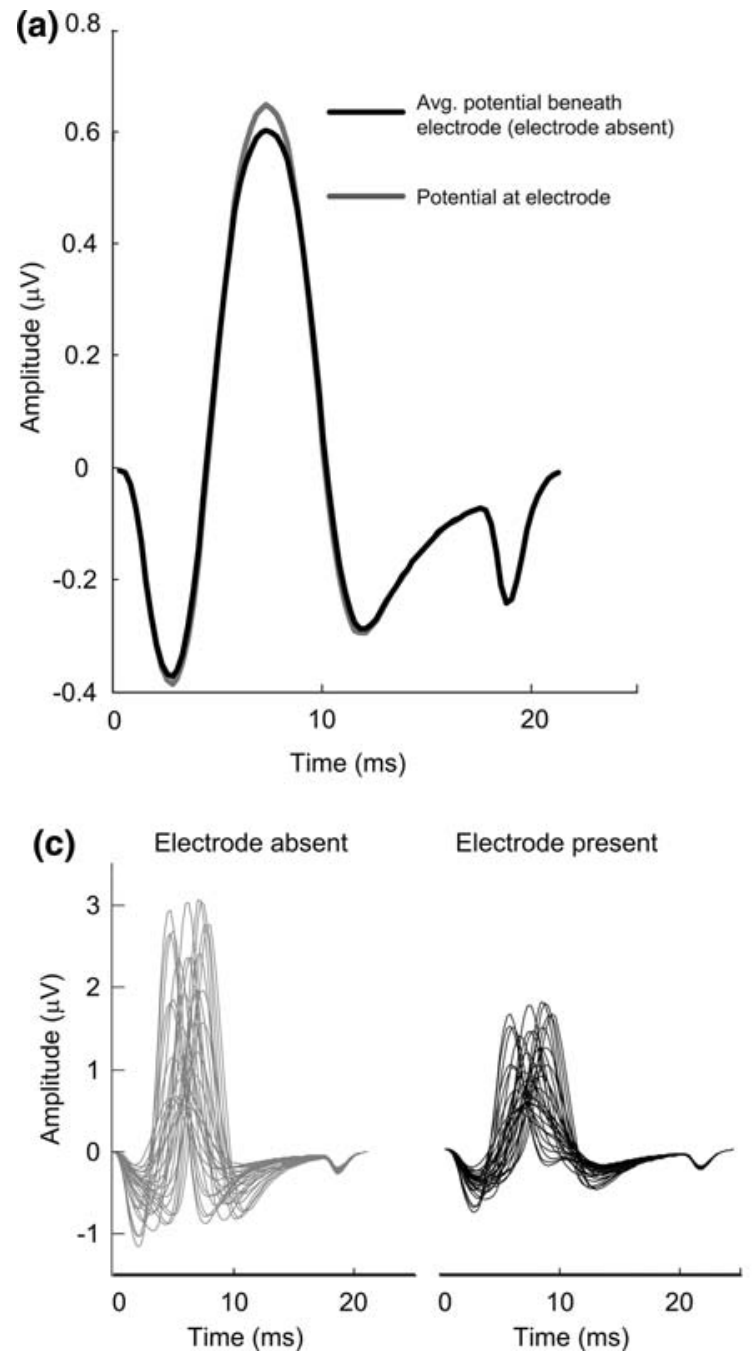

the presence of the electrode on representative action potentials detected at the skin surface beneath the electrode are compared in Fig. 5c (resistive skin) and Fig. 5d (conductive skin). In the simulated data, the presence of the electrode has a negligible effect on the potential at the skin surface in the example with a high skin conductivity $\left(R M S_{\varepsilon}=0.7 \%\right)$. In the example with a low skin conductivity (highly resistive), the influence of the electrode is slightly larger, yielding an RMS error of $5.5 \%$.

The variation in RMS error with the impedance of the electrical double layer is presented in Fig. 6. It may be seen that assertions I and II, as presented in the Introduction, are highly dependent on both the properties of the skin and the double layer. As the impedance of the double layer is reduced, the electrode starts to contribute to the volume conduction, increasing the
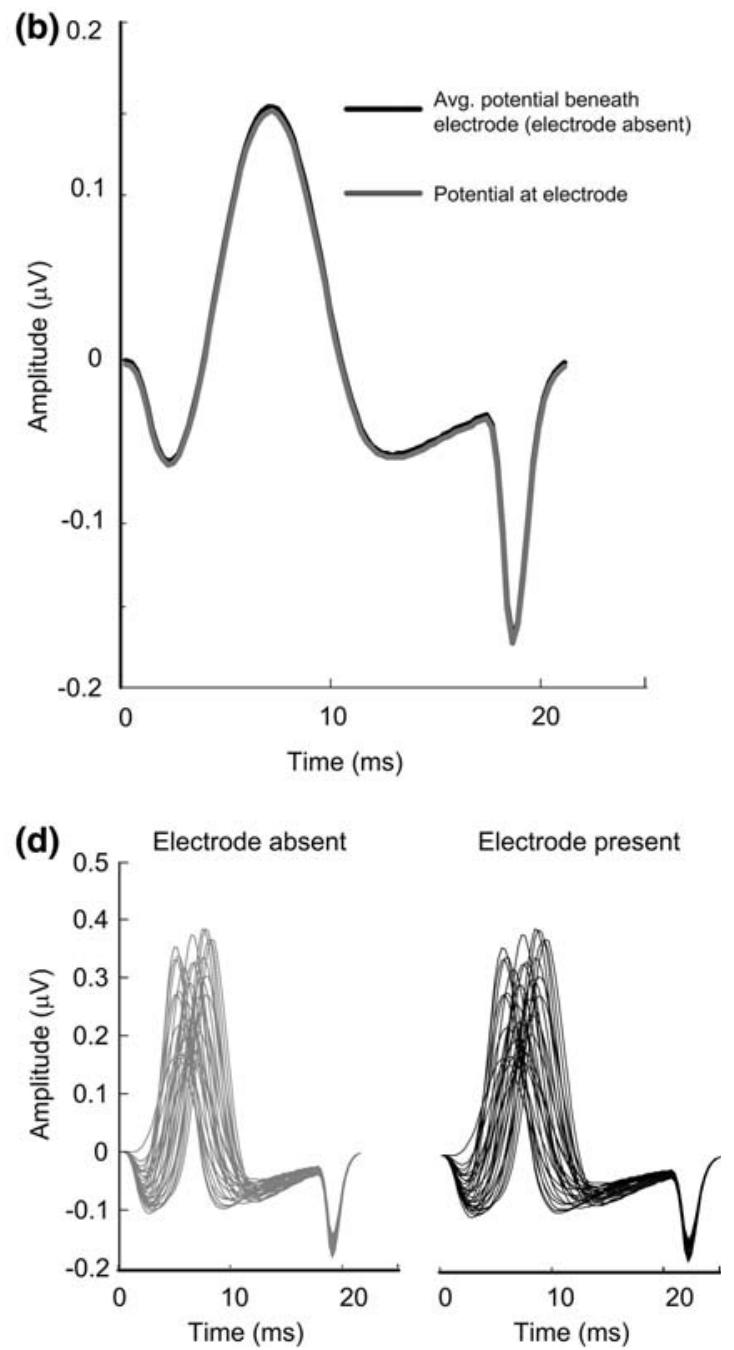

FIGURE 5. (a, b) Simulated muscle fiber action potentials detected at the electrode (black line) and average potential beneath the electrode without the electrode, including the double layer, present (gray line) for resistive (a) and conductive (b) skin. (c, d) Examples of simulated action potentials at $4 \mathrm{~mm}$ intervals along the skin surface beneath the electrode, with the electrode present and without the electrode. Data are presented for both resistive (c) and conductive skin (d). 


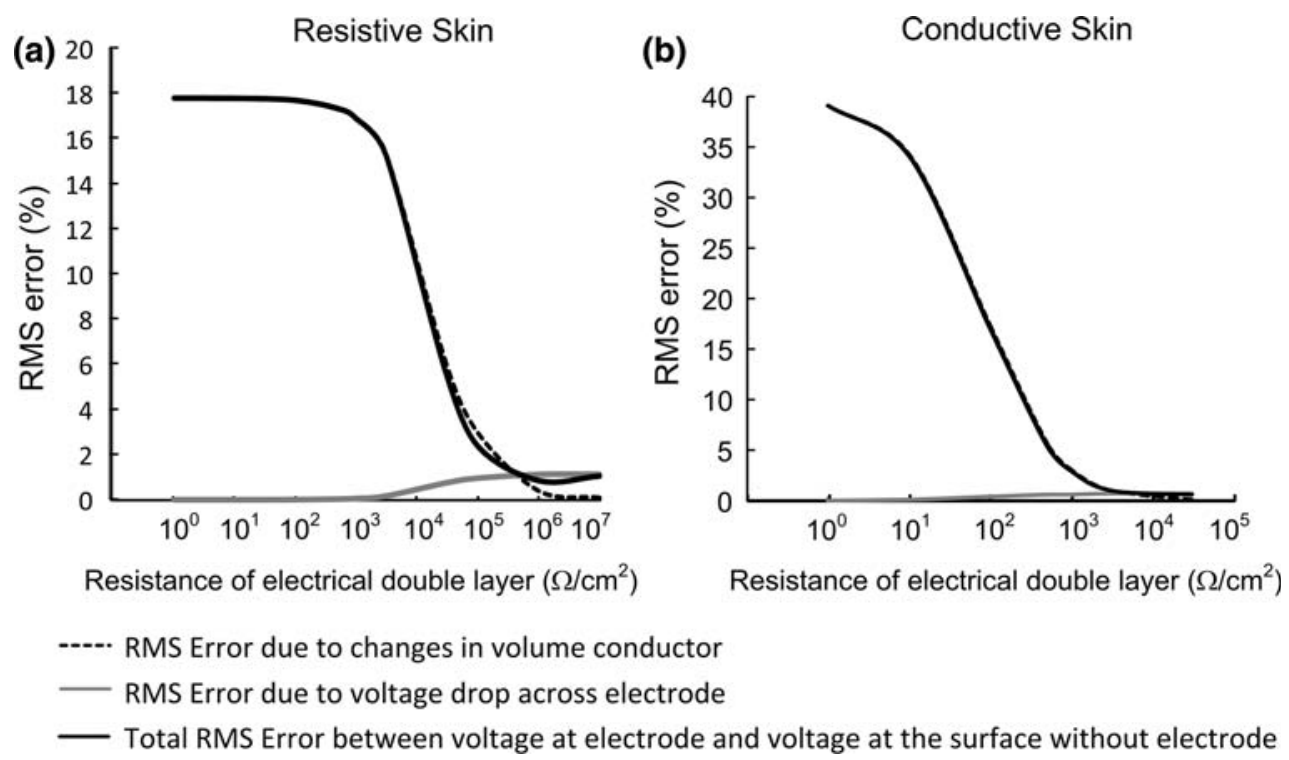

FIGURE 6. Variation in the RMS error with the impedance of the electrical double layer. The total RMS error representing the difference between the voltage recorded at the electrode and the average voltage at the skin surface when the electrode is not present is indicated with the solid black line. The error due to alterations in the volume conductor, i.e., the difference between the average voltage at the skin surface when the electrode is present and when it is not present (ref. assertion I) is shown with the dashed line. The overall difference between the potential at the electrode and the average potential directly beneath the electrode (ref. assertion II) is shown in gray. The permittivity of the electrical double layer was scaled proportional to the double layer conductivity. Data are presented for both resistive (a) and conductive (b) skin tissue.

RMS error and violating assertion I (dotted lines). This effect is most notable for the conductive skin (Fig. 6b). In the case of a high-impedance double layer, the voltage across it may become significant, challenging assertion II (gray lines). The latter effect is most notable in the case of resistive skin (Fig. 6a). Under the conditions simulated, for averaging to be precise $\left(R M S_{\varepsilon}<5 \%\right)$, the impedance of the double layer should not be lower than approximately $0.5 \mathrm{k} \Omega / \mathrm{cm}^{2}$ in the case of conductive skin, increasing to approximately $30 \mathrm{k} \Omega / \mathrm{cm}^{2}$ in the case of the resistive skin.

In Fig. 7, the relative weighting of the surface potentials by the parallel impedances expressed in Eq. (5) is examined. The data presented confirm that when there is an uneven distribution of the double layer impedances, averaging of the surface potential is indeed weighted with that distribution, even when the impedances are large enough to prohibit significant current flow between points of the electrode contact surface. This is true in the present context within an $R M S_{\varepsilon}$ of $9.4 \%$ for a relative weighting ratio of $1: 2$ and $4.3 \%$ for a relative weighting of 1:10 (Fig. 7).

\section{Experimental Results}

Large spatiotemporal variability of the experimentally obtained CMAP was also observed, as illustrated in Fig. 8. Figure 9 shows an example of the results obtained for each of the four different large electrodes for subject \#2. A high variability in amplitude across the high-density electrodes was often observed (Fig 10). In certain cases, the averaged CMAP amplitude was reduced to almost $50 \%$ of the maximal CMAP recorded at a single high-density electrode.

In total, 11 single large electrode CMAPs were obtained in this way for each subject. In most cases, the computed CMAP matched well with the CMAP obtained with the single large electrode. The mean difference expressed as the RMS error was $16.5 \%$ (range $8.6-32.8 \%$ ) for the first subject and $10.7 \%$ (range 4.4-20.1\%) for the second subject.

\section{DISCUSSION}

In this study, finite element model simulations and experimental high-density surface EMG data were used to confirm the assumption that, under practical conditions, the potential measured by a large surface EMG electrode is approximately equal to the average of the potential distribution directly beneath that electrode. Furthermore, it was shown that under the conditions examined, the application of a large electrode, which effectively connects a series of parallel lumped resistances to the skin, does not strongly affect the volume conduction properties, Figs. 5 and 9.

The simulation results confirm that both assertions I and II, defined in the Introduction, are sufficiently 
(a) Ratio of weighted electrode impedance: 1:10
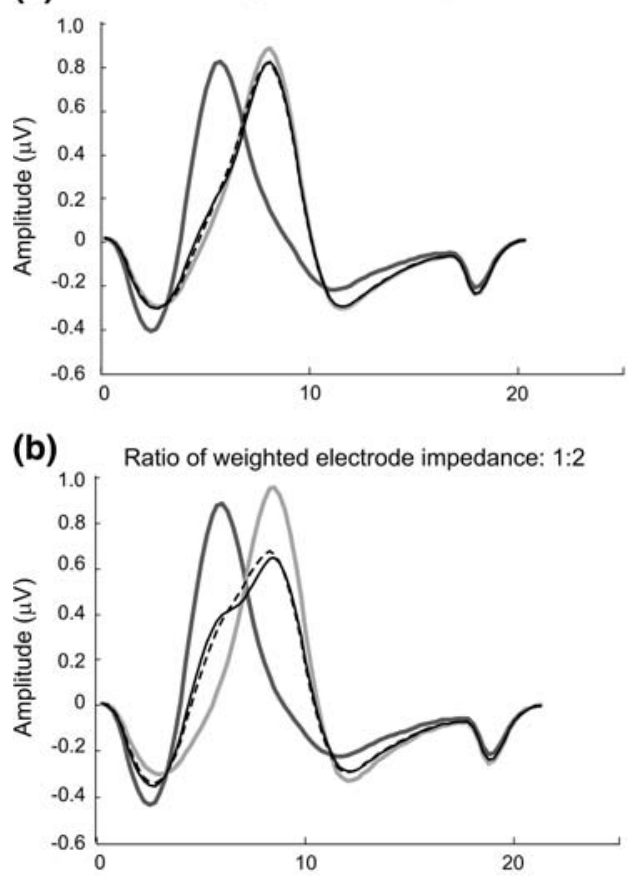

(c) Ratio of weighted electrode impedance: 1:1

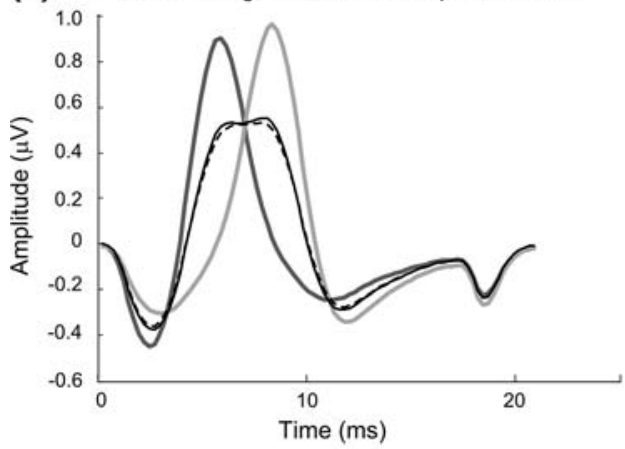

Mean potential at surface below weighted electrode impedance $A$ Mean potential at surface below weighted electrode impedance $B$ Potential at electrode

Calculated potential at electrode based on weighted average

FIGURE 7. Simulated action potentials detected at the electrode with the two sections of the electrical double layer weighted differently. The action potential at the conductive surface of the electrode is presented along with the average potential beneath each section of the electrode double layer and the action potential calculated based on relative weighting of the average potentials. The average potential calculated without taking into account the relative weighting of the action potentials by the electrode impedances is also indicated.

valid in practice when experimentally reported electrode impedance values are used. The averaging principle, although simple in its essence, is not trivial. As shown by varying the model parameters, averaging of the surface potential by the electrode does not reflect a generally valid principle of physics, but depends on the relative properties of the tissue and electrode impedances. For the range of skin properties examined, one

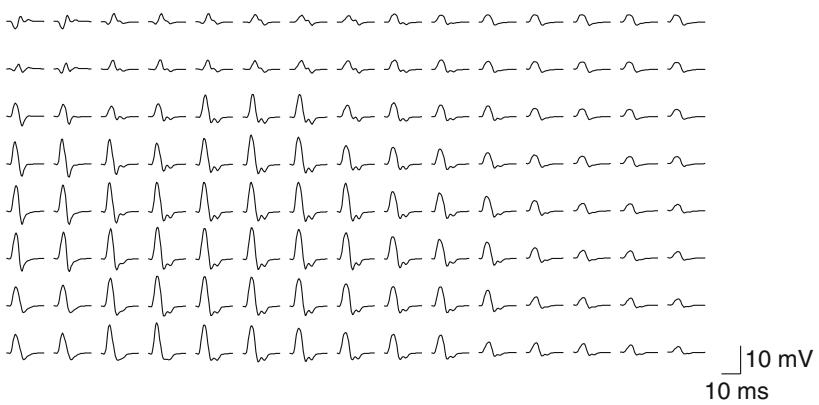

FIGURE 8. A "fingerprint" showing the spatiotemporal distribution of the CMAP response over the HD-sEMG electrode grid. The interelectrode distance in both directions is $4 \mathrm{~mm}$ resulting in an electrode size of $3 \times 5.8 \mathrm{~cm}^{2}$.

can state that the electrode-skin impedance lies well within the range where averaging is a valid concept. A range of skin conductivity values has been both reported experimentally and used in previous EMG modeling studies. To capture this range of values, two different skin conductivities were used to simulate material properties representative of resistive and conductive values within this range. A conductivity of $4.3 \times 10^{-4} \mathrm{~S} / \mathrm{m}$ was chosen for the resistive skin tissue based on the values reported by Gabriel et al. ${ }^{10}$ at $100 \mathrm{~Hz}$ and used in a previous study by Lowery et al. ${ }^{15}$ For the conductive skin tissue, a much higher conductivity of $1 \mathrm{~S} / \mathrm{m}$ was used based on the study by Roeleveld et al. ${ }^{16}$ in which skin conductivity values were estimated based on comparison of simulated and experimentally recorded EMG data.

The frequency characteristics of the (bio)electric source can also play a role due to capacitive components in both the tissue and electrode-skin interface. While at the frequencies of interest for surface EMG, the electrode-skin interface and the tissue impedances are largely dominated by the resistive components, at much higher frequencies of artificial source signals, for example in impedance tomography (e.g., $50 \mathrm{kHz}$ ), the capacitive properties of the interface and the tissue will play a significantly larger role. ${ }^{13,19}$

Finite element analysis is suited to complex geometries for which analytical solutions are not readily available. It, thus, enables the influence of the conducting electrode and electrical double layer to be explicitly included. However, there are limitations to the model which should be considered. The complex electrode-tissue interface was approximated as a single high-impedance layer of uniform thickness and electrical properties. To facilitate generation of the finite element mesh and reduce the computational burden, the thickness of the electrical double layer was increased to a value of $1 \mathrm{~mm}$, several orders of magnitude above its true value. To compensate for this, the impedance of the double layer was scaled such 
(a)

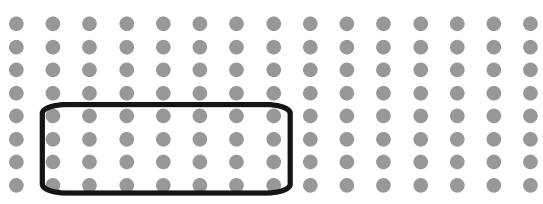

(b)

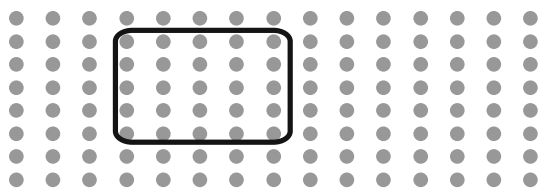

(c)

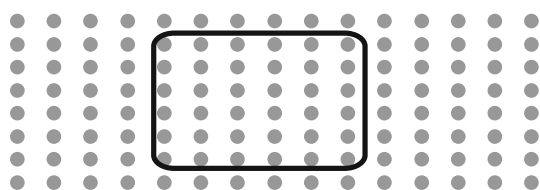

(d)

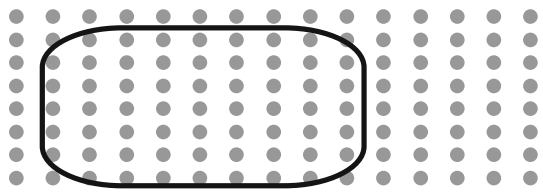

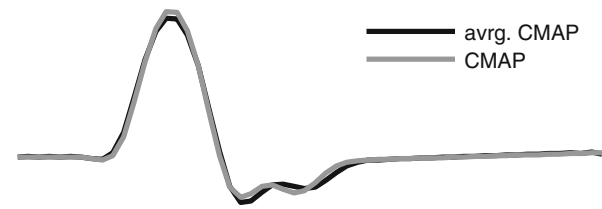
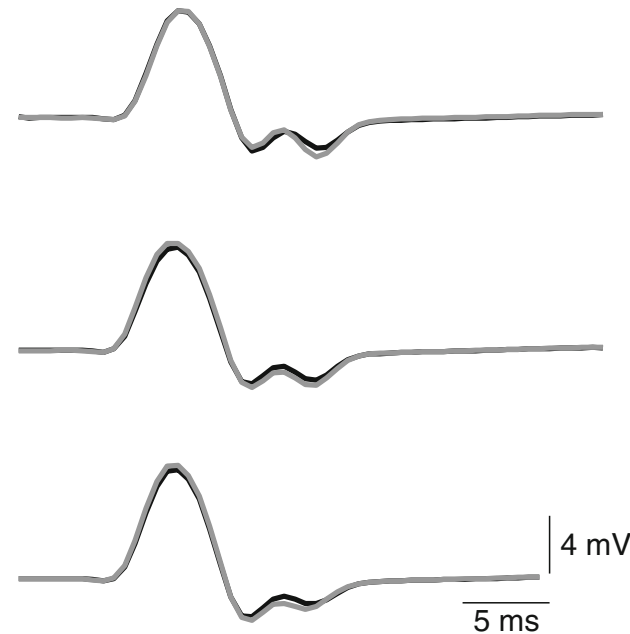

FIGURE 9. At the right side the averaged CMAPs (gray lines) as recorded by the HD-sEMG grid over the different surfaces as indicated in the left column. The electrodes in (a-d) are, respectively, $1 \times 2.6,1.8 \times 1.8,2.2 \times 2.2$, and $2.6 \times 3.4 \mathrm{~cm}^{2}$ in size. Black lines show the CMAPs as measured with large electrodes over the skin at the same positions. RMS error for (a-d) was 7.1, 7, 7.9, and $7.1 \%$, respectively.
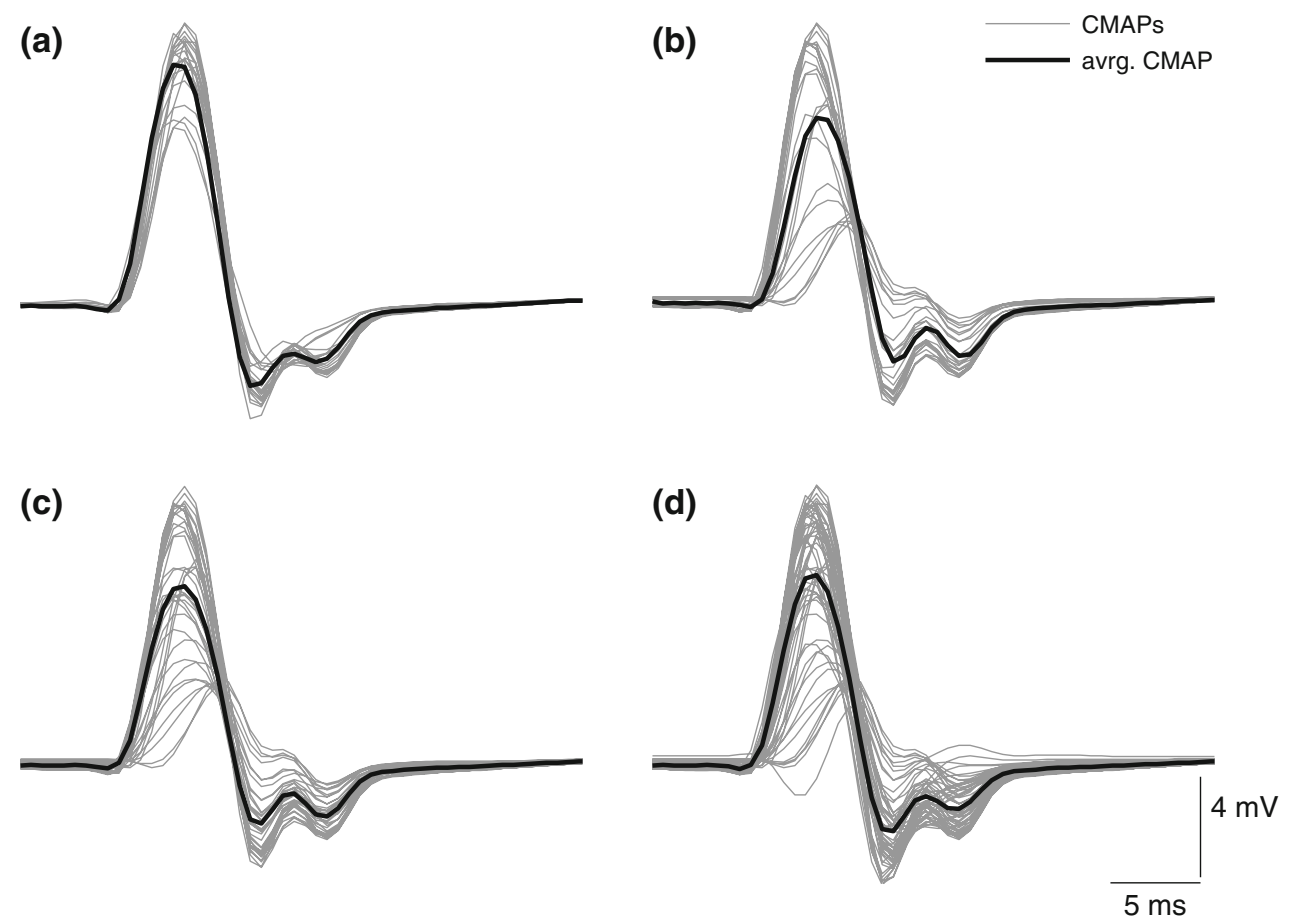

FIGURE 10. CMAP recorded using high-density surface EMG at the locations shown in Fig. 9 are presented to illustrate the high level of variability present underneath the large electrode. The mean CMAP obtained using the high-density electrode is shown in gray.

that its total impedance was not altered and remained typical of experimentally reported values. Considerable uncertainty still surrounds the choice of the most appropriate tissue electrical properties. While many studies have sought to characterize the electrical properties of muscle, skin, and fat tissue, there is 
considerable variability among the values reported in the literature. ${ }^{10}$ Furthermore, skin, which was approximated here by a homogenous tissue layer, is actually laminar in structure, with most of the impedance due to the highly resistive stratum corneum which becomes less resistive with increasing depth. Finally, to accurately capture the average potential at the skin surface, the data should be sampled at a sufficiently high spatial resolution. In the simulated data presented, the potential at the skin surface was first interpolated before averaging to increase the resolution over the surface of the skin. Increasing the distance between observation points would reduce the amplitude of average surface potential, causing it to be underestimated.

In the model, we could also have incorporated a finite layer of conductive gel. Such a layer may influence the volume conductor and its effect will again strongly depend on the conductivity of the skin. The additional error introduced will be smaller for the conductive skin than for the resistive skin, since the conductivity of electrode gel is low $(0.02-0.1 \mathrm{~S} / \mathrm{m})^{24}$ compared to the conductive skin but relatively high for the resistive skin. However, the complex electrical properties of the skin are uncertain and have been simplified within the model. Furthermore, the experimental results indicate that the application of a pregelled electrode does not strongly alter the volume conductor properties, which is consistent with the more conductive skin in the model.

High-density surface EMG was chosen to experimentally evaluate the theoretical assumptions because it changes the scheme illustrated in Fig. 1 such that each of the currents $i_{k} \approx 0$ rather than $\sum_{k=1}^{n} i_{k}=$ $i_{a} \approx 0$. Each small electrode is amplified by its own high-input impedance amplifier. The currents $i_{k}$, therefore, are negligible and, critically, they cannot emerge from the tissue at one site of the electrode and return at another site, which is essential for the principle of averaging over the skin surface (Eqs. 1 and 2). Furthermore, it should be noted that a very high electrode impedance will violate the assumption that the current flowing through the input impedance of the amplifier $i_{a}$ is much smaller than each of the components $i_{k}$.

Additional possible sources of error in the experimental data include inhomogeneities in the impedance distribution of the electrode-skin interface (the effect as presented in the simulation study, Fig. 7), some inaccuracy in the electrode placement and represented size, and variability in CMAP responses.

Our experimental results confirm the usability of the smart "branched" electrode with two simply connected, equally weighed separate parts as presented by Van Vugt and Van Dijk. ${ }^{3,23}$ Implicitly, the electrode construction is dependent on the validity of the averaging principle. We suggest that the assumption of a homogeneous distribution of electrode impedance, independent of the absolute value range, may pose the most relevant challenge to the averaging notion in general. In practice, therefore, care should be taken that electrodes used make firm and even contact to avoid these unbalanced impedances.

A wide range of possible surface EMG signals has been considered from single fiber to whole muscle activity. All these examples support the hypothesis of electrode averaging with a level of precision that is sufficient for practical purposes. It is important to consider whether there are other experimental situations in electrophysiology in which the validity of the averaging principle can be questioned? For instance, in needle EMG, higher signal frequencies are present, however, the detection surface of the electrode is generally very small. If macro-EMG recordings and needle shaft reference recording in concentric needles are considered, in the latter, modeling provides evidence for the absence of significant current flowing into and out of the electrode shaft. ${ }^{20}$ In related electrophysiological signals such as EEG and ECG, the frequency content of the signal and spatial variability are lower than in EMG. The electrodes used there are certainly relatively small compared to the variability of the spatial potential. Therefore, for these signals, one can assume with confidence that averaging over the electrode will not be significantly violated.

In conclusion, the simulation and experimental results presented here confirm the intuitive assumption that a large surface EMG electrode measures the average value of the potential over the skin beneath it. The precision of this statement appears to be sufficient for all practical EMG situations that one may conceive.

\section{ACKNOWLEDGMENTS}

This work was supported by the Prinses Beatrix Foundation (Grant No. MAR 03-0102). Dr. Thom Oostendorp is acknowledged for fruitful discussion on the modeling results.

\section{OPEN ACCESS}

This article is distributed under the terms of the Creative Commons Attribution Noncommercial License which permits any noncommercial use, distribution, and reproduction in any medium, provided the original author(s) and source are credited. 


\section{REFERENCES}

${ }^{1}$ Blok, J. H., J. P. van Dijk, G. Drost, M. J. Zwarts, and D. F. Stegeman. A high-density multichannel surface electromyography system for the characterization of single motor units. Rev. Sci. Instrum. 73:1887-1897, 2002. doi: $10.1063 / 1.1455134$

${ }^{2}$ Cantrell, D. R., S. Inayat, A. Taflove, R. S. Ruoff, and J. B. Troy. Incorporation of the electrode-electrolyte interface into finite-element models of metal microelectrodes. J. Neural Eng. 5:54-67, 2008. doi:10.1088/17412560/5/1/006

${ }^{3}$ Christova, P., A. Kossev, I. Kristev, and V. Chichov. Surface EMG recorded by branched electrodes during sustained muscle activity. J. Electromyogr. Kinesiol. 9:263276, 1999. doi:10.1016/S1050-6411(98)00048-0.

${ }^{4}$ Dimitrov, G. V., C. Disselhorst-Klug, N. A. Dimitrova, A. Trachterna, and G. Rau. The presence of unknown layer of skin and fat is an obstacle to a correct estimation of the motor unit size from surface detected potentials. Electromyogr. Clin. Neurophysiol. 42:231-241, 2002.

${ }^{5}$ Dimitrova, N. A., A. G. Dimitrov, and G. V. Dimitrov. Calculation of extracellular potentials produced by an inclined muscle fibre at a rectangular plate electrode. Med. Eng. Phys. 21:583-588, 1999. doi:10.1016/S1350-4533(99) 00087-9.

${ }^{6}$ Dumitru, D., A. A. Amato, and M. J. Zwarts. Electrodiagnostic Medicine. Philadelphia: Hanley \& Belfus, 2001.

${ }^{7}$ Farina, D., and R. Merletti. A novel approach for precise simulation of the EMG signal detected by surface electrodes. IEEE. Trans. Biomed. Eng. 48:637-646, 2001. doi: $10.1109 / 10.923782$.

${ }^{8}$ Farina, D., and R. Merletti. Effect of electrode shape on spectral features of surface detected motor unit action potentials. Acta Physiol. Pharmacol. Bulg. 26:63-66, 2001. ${ }^{9}$ Fuglevand, A. J., D. A. Winter, A. E. Patla, and D. Stashuk. Detection of motor unit action potentials with surface electrodes: influence of electrode size and spacing. Biol. Cybern. 67:143-153, 1992. doi:10.1007/BF0020 1021.

${ }^{10}$ Gabriel, C., S. Gabriel, and E. Corthout. The dielectric properties of biological tissues: I. Literature survey. Phys. Med. Biol. 41:2231-2249, 1996. doi:10.1088/0031-9155/41/11/001.

${ }^{11}$ Griep, P. A., K. L. Boon, and D. F. Stegeman. A study of the motor unit action potential by means of computer simulation. Biol. Cybern. 30:221-230, 1978. doi:10.1007/ BF00361043.

${ }^{12}$ Helal, J. N., and P. Bouissou. The spatial integration effect of surface electrode detecting myoelectric signal. IEEE Trans. Biomed. Eng. 39:1161-1167, 1992. doi:10.1109/10.168695.
${ }^{13}$ Hua, P., E. J. Woo, J. G. Webster, and W. J. Tompkins. Finite element modeling of electrode-skin contact impedance in electrical impedance tomography. IEEE Trans. Biomed. Eng. 40:335-343, 1993. doi:10.1109/10.222326.

${ }^{14}$ Lapatki, B. G., J. P. van Dijk, I. E. Jonas, M. J. Zwarts, and D. F. Stegeman. A thin, flexible multielectrode grid for high-density surface EMG. J. Appl. Physiol. 96:327-336, 2004. doi:10.1152/japplphysiol.00521.2003.

${ }^{15}$ Lowery, M. M., N. S. Stoykov, J. P. Dewald, and T. A. Kuiken. Volume conduction in an anatomically based surface EMG model. IEEE Trans. Biomed. Eng. 51:21382147, 2004. doi:10.1109/TBME.2004.836494.

${ }^{16}$ Roeleveld, K., J. H. Blok, D. F. Stegeman, and A. Van Oosterom. Volume conduction models for surface EMG; confrontation with measurements. J. Electromyogr. Kinesiol. 7:221-232, 1997. doi:10.1016/S1050-6411(97)00009-6.

${ }^{17}$ Rosell, J., J. Colominas, P. Riu, R. Pallas-Areny, and J. G. Webster. Skin impedance from $1 \mathrm{~Hz}$ to $1 \mathrm{MHz}$. IEEE Trans. Biomed. Eng. 35:649-651, 1988. doi:10.1109/ 10.4599 .

${ }^{18}$ Rosenfalck, P. Intra- and extracellular potential fields of active nerve and muscle fibres. A physico-mathematical analysis of different models. Thromb. Diath. Haemorrh. Suppl. 321:1-168, 1969.

${ }^{19}$ Rutkove, S. B., R. Aaron, and C. A. Shiffman. Localized bioimpedance analysis in the evaluation of neuromuscular disease. Muscle Nerve 25:390-397, 2002. doi:10.1002/mus. 10048 .

${ }^{20}$ Stegeman, D. F., T. H. Gootzen, M. M. Theeuwen, and H. J. Vingerhoets. Intramuscular potential changes caused by the presence of the recording EMG needle electrode. Electroencephalogr. Clin. Neurophysiol. 93:81-90, 1994. doi: 10.1016/0168-5597(94)90070-1.

${ }^{21}$ Tjon, A. T. A., H. H. Lemkes, A. J. van der Kamp-Huyts, and J. G. van Dijk. Large electrodes improve nerve conduction repeatability in controls as well as in patients with diabetic neuropathy. Muscle Nerve 19:689-695, 1996. doi: 10.1002/(SICI)1097-4598(199606)19:6 < 689::AID-MUS1 > 3.0.CO;2-6

${ }^{22}$ van Dijk, J. G., I. van Benten, C. G. Kramer, and D. F. Stegeman. CMAP amplitude cartography of muscles innervated by the median, ulnar, peroneal, and tibial nerves. Muscle Nerve 22:378-389, 1999.

${ }^{23}$ Van Vugt, J. P., and J. G. van Dijk. A convenient method to reduce crosstalk in surface EMG. Cobb Award-winning article, 2001. Clin. Neurophysiol. 112:583-592, 2001. doi: 10.1016/S1388-2457(01)00482-5.

${ }^{24}$ Webster, J. G. and L. E. Baker. Principles of Applied Biomedical Instrumentation, 3rd edn. New York: Wiley, pp. 315-316, 367-369, 1989. 